Research Article

\title{
A New Approach for Solving Rub-Impact Dynamic Characteristics of Shrouded Blades Based on Macroslip Friction Model
}

\author{
Guofang Nan $\mathbb{D}^{1},{ }^{1}$ Jianyang Lou, ${ }^{1}$ Chuanchong Song, ${ }^{1}$ and Min Tang ${ }^{2}$ \\ ${ }^{1}$ School of Energy and Power Engineering, University of Shanghai for Science and Technology, Shanghai 200093, China \\ ${ }^{2}$ Shanghai Electric Gas Turbine Co., Ltd., Shanghai 200240, China \\ Correspondence should be addressed to Guofang Nan; ngf@usst.edu.cn
}

Received 30 September 2019; Accepted 21 December 2019; Published 14 January 2020

Academic Editor: Nuno M. Maia

Copyright (C) 2020 Guofang Nan et al. This is an open access article distributed under the Creative Commons Attribution License, which permits unrestricted use, distribution, and reproduction in any medium, provided the original work is properly cited.

Based on the macroslip friction model, a new dynamic model of the shrouded blades for rotating machinery is developed to study the impact vibration between the adjacent blades. Unlike the traditional analytical method of the shrouded blade based on the simple Coulomb friction model, a new approach is developed that the macroslip friction model is used to represent a more accurate rubbing behavior (more closer to reality) between the shrouds. By means of the harmonic balance method, the friction force and the normal pressure are translated into the equivalent stiffness and the equivalent damping. The Galerkin method is adopted to reduce the dimension of the equation to obtain the 1-DOF equation of motion, and the dynamic response of the shrouded blade is solved by Runge-Kutta numerical method. The effects of parameters such as the gap of shrouds, the mass of the tip, the contact angle, and the normal stiffness between the shrouded blades on the damping characteristics are discussed. The results show that the gap of tips has a significant effect on the vibration amplitude of the blade. Within a certain range, with the decrease of the gap, the amplitude of the blade tip is getting smaller while the resonant speed is increasing. The mass of the shroud has little effect on the damping characteristics, while the contact angle has a great influence on the equivalent stiffness and damping. Increasing the contact angle to a certain extent can effectively reduce the vibration amplitude of the blade, and the normal contact stiffness also has an important influence in reducing the vibration. The research results based on the new method in this paper are compared with the published articles and agree well. The research work is important to the accurate calculations and design and control of the shrouded blades for rotating machinery.

\section{Introduction}

The shrouded blades are widely used in the steam turbine for it can reduce the energy dissipated by collision and friction between the shrouds. At present, in the study of the dynamics and control for the shrouded blades, the classical Coulomb friction theory is widely adopted to describe the friction behaviors between the shrouds $[1,2]$. The researchers $[3,4]$ reported that the classical Coulomb friction theory has some limitations and cannot accurately describe the microscopic motion between the contact surfaces. The collision and friction between shrouds contain a variety of states such as adhesion, slip, and separation, which characterize strong nonlinear characteristics. Due to the complex nonlinear characteristics, the principle research on the vibration damping is still in the exploratory stage and the dynamic response of the system cannot be predicted $[5,6]$.

The theory is abundant in the analysis of the contactimpact damping between shrouds. Menq et al. [7] studied the variation of normal pressure and proposed a friction model which can be used to analyze the existence of adhesion, slip, and separation between shrouds. Mindlin et al. [8] and Metherell and Dille [9] proposed a microslip friction model. Based on the Coulomb friction model, Nan et al. $[10,11]$ established the 2-DOF system of damping blades, and the influence of different parameters on the dynamic response of the system is studied by using the numerical integration method. Cao et al. [12] simplified the rotor blade with a 
rotational cantilever beam structure and analyzed the nonlinear vibration characteristics of the blade applying the Coulomb friction model. Lu et al. [13] built an equivalent model of the contact-impact shrouds with the clearance. In recent years, many scholars used the finite element contact theory to study the dynamic characteristics of damping blades. Xie and Zhang [14] developed a three-dimensional finite element analysis model of shrouded blades based on the macroslip model and the harmonic balance method. Szwedowicz et al. [15] analyzed the damping performance for the different contact configurations between the shrouds numerically. Mohammad et al. [16] established an analytical expression to compute the Jacobian matrix for 3D friction contact. Ma et al. [17-19] carried out much research and made a lot of progress on the dynamic analysis of the shrouded blades and the rotor blade. Ma et al. [17] established a dynamic model of shrouded blades with impacts among adjacent blades under consideration of the centrifugal stiffening, the spin softening, and Coriolis force. Hou et al. [20] focused on the complex nonlinear behaviors of an aircraft rub-impact rotor system based on the harmonic balance method combined with the alternating frequency/ time-domain procedure (HB-AFT method).

The authors simplified the shrouded blade as a rotating cantilever beam with a lumped mass to investigate the dynamic behavior of the system. The most innovative work in this study is that researchers applied the macroslip model which can accurately describe the adhesion, the slip, and the separation states and considered the different contact states and the transition mechanism. A hysteresis loop that can approximately reflect the state of contact surfaces is developed. The friction force and the normal pressure are translated into the equivalent stiffness and the equivalent damping by means of the harmonic balance method [1,21,22]. Finally, the effect of the structural parameters on the dynamic responses is analyzed and the important conclusions are drawn. The results based on the new approach are compared with the related references and agree well.

\section{Mathematical Model}

2.1. The Contact-Impact Dynamic Model. The complex coupling motion often exists between the two contact surfaces of the shrouds during operation. In order to study the complicated dynamic response, a more accurate model will be established in this section. It is assumed that the stress of the contact surface is uniform, and the shroud-to-shroud contact is a type of the point contact. The states of the neighbouring contact surface, including adhesion, slip, and separation, are dependent on the tangential and normal relative displacements. In order to study conveniently, the normal force is assumed to vary linearly with the relative displacement of the contact surface, and the tangential friction force has typical nonlinear characteristics. The structural parameters and the interaction forces between the shrouds are shown in Figure 1. The gap between the shrouds is $d$, and the contact angle is $\gamma$. An assumption is proposed that the relative motion between the shrouds is a simple harmonic motion, and the relative displacement of the main vibration direction of the contact surface $v$ can be expressed as

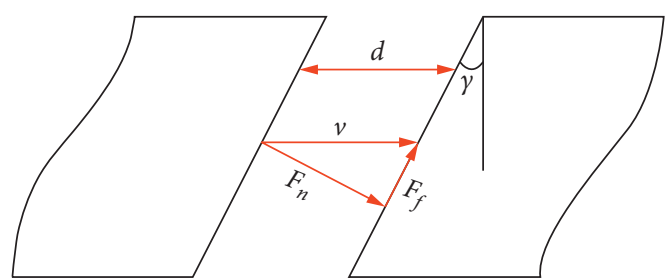

Figure 1: Schematic diagram of structural parameters and interaction force between shrouds.

$$
v=B \cos (\omega t-\varphi)=B \cos \theta,
$$

where $B$ is the amplitude of the steady-state vibration, $\omega$ is the frequency of exciting force, and $\phi$ is the phase angle.

The tangential relative displacement of the contact surface $\Delta u$ is given by the following equation:

$$
\Delta u=v \sin \gamma=B \sin \gamma \cos \theta .
$$

The normal force of the contact surface $F_{n}$ can be expressed by the piecewise linear function:

$$
F_{n}= \begin{cases}k_{n}(v(l)-d) \cos \gamma, & v(l)>d, \\ 0, & -d \leq v(l) \leq d, \\ k_{n}(v(l)+d) \cos \gamma, & v(l)<-d,\end{cases}
$$

where $k_{n}$ represents the normal contact stiffness between the shrouds.

When the macroslip model is adopted to substitute for the traditional simple Coulomb friction model for the shrouded blades' friction dynamics, the hysteretic loops of friction force $F_{f}$ varies with the relative displacement $\Delta u$, shown in Figure 2. There is an initial gap between the neighbouring shrouds for this type of blades. The vibration of blade starts from the point $A(\theta=0)$ where the left contact surface is at the maximum positive displacement, and then the left contact surface arrives at the point $B\left(\theta=\theta_{1}\right)$ along the line $A B$. From the point $B$ to the point $C\left(\theta=\theta_{2}\right)$, the two contact surfaces begin to slide towards the opposite direction (based on this assumption, the friction force remains constant). The separation of surface and surface occurs at the point $C$; the friction force reduces to zero at the point $D$. The two corresponding rubbing surfaces move along the line $D D^{\prime}$ in the opposite direction from the point $D$ to $D^{\prime}$ $\left(\theta=\pi-\theta_{2}\right)$, where the right surface is under the state of contact, and then the right surface arrives at the point $E^{\prime}$ $\left(\theta=\theta_{3}\right)$ along the line $D^{\prime} E^{\prime}$, where the two contact surfaces are in a state of adhesion. After that, the contact surface slides to the maximum negative displacement point $A^{\prime}$ $(\theta=\pi)$. The right contact surface moves from the adhesive state $A^{\prime} B^{\prime}$ (point $B^{\prime}: \theta=\pi+\theta_{1}$ ) to the sliding state $B^{\prime} C^{\prime}$, and the separation occurs at the point $C^{\prime}\left(\theta=\pi+\theta_{2}\right)$. Then, the right contact surface gets to the point $D$ along the line $D^{\prime} D$, and the left contact surface reaches the point $E\left(\theta=\pi+\theta_{3}\right)$ along the line $D E$, where it is in the state of adhesion. At last, the left contact surface slides to the point $A$. So far, a cycle for the vibration of the system with the impact and friction has been completed. 


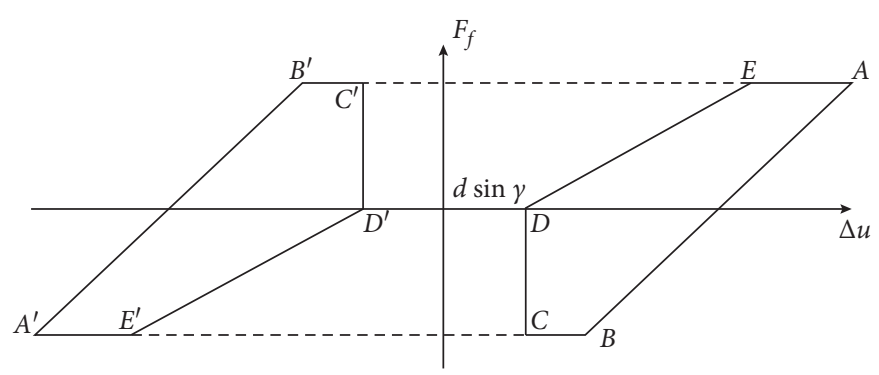

Figure 2: Hysteretic loops of friction force vs relative displacement.

The tangential friction force between the shroud contact surfaces $F_{f}$ is

$$
F_{f}= \begin{cases}k_{d}(\Delta u-B \sin \gamma)+\mu k_{n} \cos \gamma(v-d), & 0 \leq \theta<\theta_{1}, \\ -\mu k_{n} \cos \gamma(B-d), & \theta_{1} \leq \theta<\theta_{2}, \\ 0, & \theta_{2} \leq \theta<\pi-\theta_{2}, \\ k_{d}(\Delta u+d \sin \gamma), & \pi-\theta_{2} \leq \theta<\theta_{3}, \\ -\mu k_{n} \cos \gamma(B-d), & \theta_{3} \leq \theta<\pi, \\ k_{d}(\Delta u+B \sin \gamma)+\mu k_{n} \cos \gamma(v+d), & \pi \leq \theta<\pi+\theta_{1}, \\ \mu k_{n} \cos \gamma(B-d), & \pi+\theta_{1} \leq \theta<\pi+\theta_{2}, \\ 0, & \pi+\theta_{2} \leq \theta<2 \pi-\theta_{2}, \\ k_{d}(\Delta u-d \sin \gamma), & 2 \pi-\theta_{2} \leq \theta<\pi+\theta_{3}, \\ \mu k_{n} \cos \gamma(B-d), & \pi+\theta_{3} \leq \theta \leq 2 \pi,\end{cases}
$$

where

$$
\begin{aligned}
& \theta_{1}=\cos ^{-1}\left(\frac{k_{d} B \tan \gamma+2 \mu k_{n} d-\mu k_{n} B}{k_{d} B \tan \gamma+\mu k_{n} B}\right), \\
& \theta_{2}=\cos ^{-1}\left(\frac{d}{B}\right), \\
& \theta_{3}=\cos ^{-1}\left[-\frac{d k_{d} \tan \gamma+\mu k_{n}(B-d)}{B k_{d} \tan \gamma}\right],
\end{aligned}
$$

where $k_{d}$ is the shear stiffness of the contact surface and $\mu$ is the friction coefficient.

The harmonic balance method (HBM) is adopted to linearize the nonlinear friction force (the piecewise linear characteristics of the system are essential nonlinear) to obtain the equivalent stiffness and the equivalent damping. The tangential friction force between the shroud contact surfaces $F_{f}$ can be expressed by

$$
F_{f}=F_{k} \cos \theta+F_{c} \sin \theta=f_{k} \Delta u+f_{c} \frac{\mathrm{d} \Delta u}{\mathrm{~d} t}
$$

where

$$
\begin{aligned}
f_{k}= & \frac{F_{k}}{B}=\frac{1}{\pi B} \int_{0}^{2 \pi} F_{f} \cos \theta \mathrm{d} \theta=\frac{2}{\pi B}\left\{\frac { 1 } { 4 } B k _ { d } \operatorname { s i n } \gamma \left(2 \theta_{1}\right.\right. \\
& \left.+\sin \left(2 \theta_{1}\right)-4 \sin \theta_{1}\right)+\frac{1}{4} \mu k_{n} \cos \gamma\left(2 B \theta_{1}+B \sin \left(2 \theta_{1}\right)\right. \\
& \left.-4 d \sin \theta_{1}\right)-\mu k_{n} \cos \gamma(B-d) \times\left(\sin \theta_{2}-\sin \theta_{1}\right. \\
& \left.-\sin \theta_{3}\right)+\frac{1}{4} k_{d} \sin \gamma\left[2 B\left(\theta_{2}+\theta_{3}-\pi\right)+B\left(\sin \left(2 \theta_{3}\right)\right.\right. \\
& \left.\left.\left.+\sin \left(2 \theta_{2}\right)\right)+4 d\left(\sin \theta_{3}-\sin \theta_{2}\right)\right]\right\}, \\
f_{c}= & \frac{F_{c}}{-B \omega}=-\frac{1}{B \omega \pi} \int_{0}^{2 \pi} F_{f} \sin \theta \mathrm{d} \theta=-\frac{2}{B \omega \pi} \\
& \cdot\left[\frac{1}{4} B k_{d} \sin \gamma\left(\cos \theta_{1}-\cos \left(2 \theta_{1}\right)-3\right)+\frac{1}{4} \mu k_{n} \cos \gamma(B\right. \\
& \left.+4 d \cos \theta_{1}-B \cos \left(2 \theta_{1}\right)-4 d\right)+\mu k_{n} \cos \gamma(B-d) \\
& \cdot\left(\cos \theta_{2}-\cos \theta_{1}\right)+\frac{1}{4} k_{d} \sin \gamma\left(B \cos \left(2 \theta_{2}\right)-B \cos \left(2 \theta_{3}\right)\right. \\
& \left.\left.-4 d \cos \theta_{3}-4 d \cos \theta_{2}\right)-\mu k_{n} \cos \gamma(B-d)\left(1+\cos \theta_{3}\right)\right] .
\end{aligned}
$$

Similarly, the normal force $F_{n}$ on the contact surface can be obtained:

$$
F_{n}=P_{k} \cos \theta+P_{c} \sin \theta=p_{k} \Delta u+p_{c} \frac{\mathrm{d} \Delta u}{\mathrm{~d} t},
$$

where

$$
\begin{aligned}
p_{k}= & \frac{P_{k}}{B}=\frac{1}{\pi B} \int_{0}^{2 \pi} F_{n} \cos \theta \mathrm{d} \theta=\frac{1}{\pi B}\left[k _ { n } \operatorname { c o s } \gamma \left(2 B \theta_{2}\right.\right. \\
& \left.\left.+B \sin \left(2 \theta_{2}\right)-4 d \sin \theta_{2}\right)\right], \\
p_{c}= & \frac{P_{c}}{-B \omega}=-\frac{1}{B \omega \pi} \int_{0}^{2 \pi} F_{n} \sin \theta \mathrm{d} \theta=0,
\end{aligned}
$$

where $F_{k}$ and $F_{c}$ represent the Fourier expansion coefficients of $F_{f}$, respectively; $P_{k}$ and $P_{c}$ denote the Fourier expansion coefficients of $F_{n}$, respectively; $f_{k}$ and $f_{c}$ are the equivalent stiffness and the equivalent damping of $F_{f}$, respectively; $p_{k}$ and $p_{c}$ represent the equivalent stiffness and the equivalent damping of $F_{n}$, respectively.

As it has been stated above, it can be inferred that the normal force has an effect on the stiffness of the system without changing the damping of the system. Considering 
the effect of the friction force and the normal force, the equivalent stiffness $K_{\mathrm{eq}}$ and the equivalent damping $C_{\mathrm{eq}}$ can be obtained:

$$
\begin{aligned}
& K_{\mathrm{eq}}= \begin{cases}\left(f_{k}+p_{k}\right) \sin \gamma, & v>d, v<-d, \\
0, & -d \leq v \leq d,\end{cases} \\
& C_{\mathrm{eq}}= \begin{cases}\left(f_{c}+p_{c}\right) \sin \gamma, & v>d, v<-d, \\
0, & -d \leq v \leq d .\end{cases}
\end{aligned}
$$

2.2. The Governing Equations of Motion. A shrouded blade often vibrates and impacts with the adjacent blades during operation. The shrouded blade is simplified as a cantilever beam with a lumped mass $m_{0}$, the adjacent blades are simplified to be springs with the stiffness coefficient $k_{n}$, a local coordinate system is established, $x$ is the radial direction along the blade, and $y$ is the transverse direction which is perpendicular to the $x$ direction, as shown in Figure 3. Taking a differential segment of the beam with the length of $\mathrm{d} x$ and analyzing the equilibrium of the forces in the transverse direction, the equilibrium equation of the system can be obtained:

$$
\begin{aligned}
& f_{s}-f_{a} \frac{\partial v}{\partial x}-\left[f_{s}+\frac{\partial f_{s}}{\partial x} \mathrm{~d} x-f_{a} \frac{\partial v}{\partial x}-\frac{\partial\left(f_{a}(\partial v / \partial x)\right)}{\partial x} \mathrm{~d} x\right] \\
& \quad-c \frac{\partial v}{\partial t} \mathrm{~d} x+\left[f_{w}(t)-\left(F_{n} \cos \gamma+F_{f} \sin \gamma\right) \delta(x-l)\right] \mathrm{d} x \\
& =\rho A \mathrm{~d} x \frac{\partial^{2} v}{\partial t^{2}},
\end{aligned}
$$

where $v(x, t)$ is the displacement of the bending vibration, $\rho$ is the mass density, $A$ is the cross-sectional area, $l$ is the length of the blade, and $c$ is the viscous damping coefficient. $\delta(x-l)$ is the Dirac delta function which is used to describe the contact states, and it is equal to zero if $x$ is not equal to $l$. The shear force $f_{s}$ acting on the cross-section can be written as

$$
f_{s}=\frac{\partial M}{\partial x}=\frac{\partial}{\partial x}\left(\text { EI } \frac{\partial^{2} v}{\partial x^{2}}\right)
$$

where $M$ is the moment acting on the cross-section and EI is the flexural stiffness.

The radial load caused by the centrifugal force on the segment can be expressed as

$$
f_{a}=\frac{1}{2} \rho A \Omega^{2}\left(l^{2}-x^{2}\right)+m_{0} l \Omega^{2},
$$

where $m_{0}$ is the mass of the shroud and $\Omega$ is the rotating speed of the rotor.

The external periodic aerodynamic force is defined as

$$
f_{w}(t)=F_{w 0} \sin (3 \Omega t),
$$

where $F_{w 0}$ is the amplitude of the aerodynamic load.

Then, equation (14) can be rewritten as

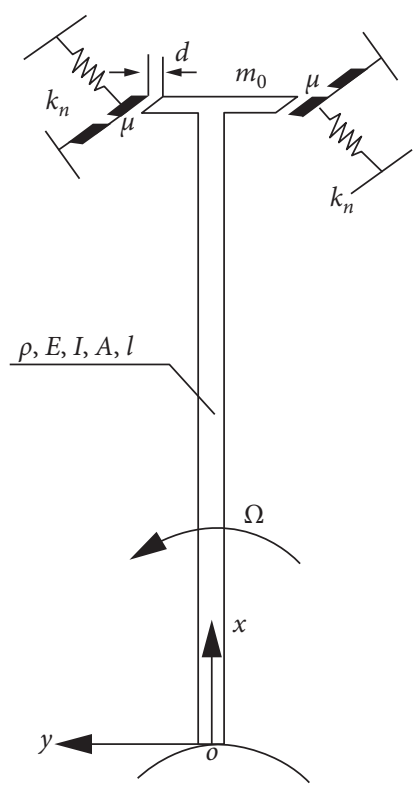

FIgURE 3: The analytical model of the shrouded blade.

$$
\begin{aligned}
& -\rho A \Omega^{2} x \frac{\partial v}{\partial x}+\left[\frac{1}{2} \rho A \Omega^{2}\left(l^{2}-x^{2}\right)+m_{0} l \Omega^{2}\right] \frac{\partial^{2} v}{\partial x^{2}} \\
& +F_{w 0} \sin (3 \Omega t)-\left(F_{N} \cos \gamma+F_{f} \sin \gamma\right) \delta(x-l)=\mathrm{EI} \frac{\partial^{4} v}{\partial x^{4}} \\
& +\rho A \frac{\partial^{2} v}{\partial t^{2}}+c \frac{\partial v}{\partial t}
\end{aligned}
$$

Applying the Galerkin method and introducing the first $m$ terms of bending modes, the displacement variable is expanded as

$$
v(x, t)=\sum_{i=1}^{m} \phi_{i}(x) q_{i}(t),
$$

where $q_{i}(t)$ is the coefficient to be determined and $\phi_{i}(x)$ is the eigenfunction of the cantilever beam, which is given by

$$
\phi_{i}(x)=\frac{\sinh \lambda_{i} l+\sin \lambda_{i} l}{\cosh \lambda_{i} l+\cos \lambda_{i} l}\left(\cosh \lambda_{i} x-\cos \lambda_{i} x\right)+\sin \lambda_{i} x-\sinh \lambda_{i} x,
$$

where $\lambda_{i} l$ are determined by the following frequency equation:

$$
1+\cos \lambda_{i} l \cosh \lambda_{i} l+\frac{m_{0}}{\rho A l} \lambda_{i} l\left(\cos \lambda_{i} l \sinh \lambda_{i} l-\sin \lambda_{i} l \cosh \lambda_{i} l\right)=0 .
$$

Substituting equation (19) into equation (18) and orthogonalizing the equation with respect to the set $\phi_{j}(x)$, one can obtain 


$$
\begin{aligned}
& \mathrm{EI} \int_{0}^{l} \phi_{j}(x) \sum \phi_{i}^{(4)}(x) q(t) \mathrm{d} x+\rho A \int_{0}^{l} \phi_{j}(x) \sum \phi_{i}(x) \ddot{q}(t) \mathrm{d} x \\
& \quad+c \int_{0}^{l} \phi_{j}(x) \sum \phi_{i}(x) \dot{q}(t) \mathrm{d} x=-\rho A \Omega^{2} \int_{0}^{l} \phi_{j}(x) \\
& \quad \cdot x \sum \phi_{i}^{\prime}(x) q(t) \mathrm{d} x+m_{0} l \Omega^{2} \int_{0}^{l} \phi_{j}(x) \sum \phi_{i}^{\prime \prime}(x) q(t) \mathrm{d} x \\
& \quad+F_{w 0} \sin 3 \Omega t \int_{0}^{l} \phi_{j}(x) \mathrm{d} x+\frac{1}{2} \rho A \Omega^{2} \int_{0}^{l} \phi_{j}(x)\left(l^{2}-x^{2}\right) \\
& \quad \cdot \sum \phi_{i}^{\prime \prime}(x) q(t) \mathrm{d} x-\left(F_{N} \cos \gamma+F_{f} \sin \gamma\right) \int_{0}^{l} \phi_{j}(x) \delta(x-l) \mathrm{d} x .
\end{aligned}
$$

When $m=1$, equation (22) can be rewritten as

$$
\begin{aligned}
& \rho A U_{1} \ddot{q}+c U_{1} \dot{q}+\left(\mathrm{EIU}_{2}+\rho A \Omega^{2} U_{3}-\frac{1}{2} \rho A \Omega^{2} U_{4}\right. \\
& \left.-m_{0} l \Omega^{2} U_{5}\right) q=u_{1} \sin (3 \Omega t)-\left(F_{N} \cos \gamma+F_{f} \sin \gamma\right) u_{2},
\end{aligned}
$$

where

$$
\begin{aligned}
& U_{1}=\int_{0}^{l} \phi_{1}^{2}(x) \mathrm{d} x, \\
& U_{2}=\int_{0}^{l} \phi_{1}^{5}(x) \mathrm{d} x, \\
& U_{3}=\int_{0}^{l} x \phi_{1}(x) \phi_{1}^{\prime}(x) \mathrm{d} x, \\
& U_{4}=\int_{0}^{l} \phi_{1}^{\prime \prime}(x) \phi_{1}(x)\left(l^{2}-x^{2}\right) \mathrm{d} x, \\
& U_{5}=\int_{0}^{l} \phi_{1}(x) \phi_{1}^{\prime \prime}(x) \mathrm{d} x, \\
& u_{1}=F_{w 0} \int_{0}^{l} \phi_{1}(x) \mathrm{d} x, \\
& u_{2}=\phi_{1}(l) .
\end{aligned}
$$

Then; the governing differential equation of motion can be formulated as

$$
M \ddot{q}+C_{0} \dot{q}+K_{0} q=\left(\frac{u_{1}}{u_{2}}\right) \sin (3 \Omega t)-\left(F_{N} \cos \gamma+F_{f} \sin \gamma\right),
$$

where

$$
\begin{aligned}
M & =\frac{\rho A U_{1}}{u_{2}}, \\
C_{0} & =\frac{c U_{1}}{u_{2}}, \\
K_{0} & =\frac{\left(\mathrm{EIU} U_{2}+\rho A \Omega^{2} U_{3}-(1 / 2) \rho A \Omega^{2} U_{4}-m_{0} l \Omega^{2} U_{5}\right)}{u_{2}} .
\end{aligned}
$$

Taking equation (12) and equation (13) into consideration, equation (25) can be rewritten as follows:

$$
M \ddot{q}+\left(C_{0}+C_{\text {eq }}\right) \dot{q}+\left(K_{0}+K_{\text {eq }}\right) q=\left(\frac{u_{1}}{u_{2}}\right) \sin (3 \Omega t) .
$$

\section{Numerical Results and Discussion}

The differential equation is solved by the Runge-Kutta method for its accuracy and stability. When solving the equation, it gives an initial value of $B$. The equivalent stiffness and damping are obtained from equation (12) and equation (13) and then substituted into equation (26) to calculate the amplitude of the dynamic responses. Based on the numerical calculation, the influence of the different structure parameters of the shrouded blades (the gap between shrouds $d$, the contact angle $\gamma$, the mass of shroud $m_{0}$, and the normal contact stiffness $k_{n}$ ) on the vibration characteristics of the system is investigated. When one parameter of the system is analyzed, the other parameters remain constant. The amplitude of aerodynamic force $F_{w 0}$ is equal to $100 \mathrm{~N}$. The ratio of the tangential shear stiffness to the normal contact stiffness $k_{d} / k_{n}$ is equal to $2(1-v) /(2-v)$. Poisson's ratio $v$ is 0.3 . The blade material constants and geometrical parameters are in accordance with those of reference [9], as shown in Table 1.

The influence of the gap between the shrouds on the dry friction damping characteristics will be studied in this section. The gap varies from $0.02 \mathrm{~mm}$ to $0.06 \mathrm{~mm}$, the contact angle between the two neighbouring shrouded blades $\gamma$ is equal to $\pi / 3$, the mass of the shroud $m_{0}$ is $0.025 \mathrm{~kg}$, and the normal contact stiffness is equal to $1 \times 10^{7} \mathrm{~N} / \mathrm{m}$. Figures 4 and 5 represent the vibration responses of the blade tip in the time domain and frequency domain at the rotational speed $n=5000 \mathrm{r} / \mathrm{min}$, respectively. It can be shown from Figure 4 that the system is basically in the steady-state response after $0.2 \mathrm{~s}$, and the maximum response amplitude is greater than that of the gap value $0.03 \mathrm{~mm}$. This indicates that the adjacent shrouds are in the state of contact with each other. From Figure 5, it can be shown that the maximum response frequency appears at $250 \mathrm{~Hz}$, which is consistent with the fundamental frequency of the aerodynamic force. The results show that the numerical calculation results are in accordance with the theory.

Figure 6 shows the effect of the different gap values on the damping characteristics. As can be seen, the value of the gap between shrouds has a significant impact on the amplitude of the vibration in the $Y$ direction. When the gap value increases from $0.02 \mathrm{~mm}$ to $0.06 \mathrm{~mm}$, the vibration amplitude of the blade increases gradually. The maximum amplitude at $d=0.06 \mathrm{~mm}$ is 3.3 times than that of at $d=0.02 \mathrm{~mm}$. At the same time, it can be seen that with the increase of the gap, the resonant rotational speed decreases. Therefore, in the design of the damping structure of the shrouded blades, the resonant speed can be avoided and the resonant amplitude can be reduced by adjusting the clearance between shrouds. 
TABle 1: Material constants and geometrical parameters for the model.

\begin{tabular}{lccc}
\hline Sign & Description & Value & Unit \\
\hline EI & Flexural stiffness & 343 & $\mathrm{~N} * \mathrm{~m}$ \\
$A$ & Cross-sectional area & $4.2 \times 10^{-4}$ & $\mathrm{~m}^{2}$ \\
$\rho$ & Mass density & 7800 & $\mathrm{~kg} / \mathrm{m}^{3}$ \\
$l$ & Length of the blade & 150 & $\mathrm{~mm}$ \\
$c$ & Viscous damping coefficient & 29.8 & $\mathrm{~N} * \mathrm{~s} / \mathrm{m}$ \\
$b$ & Width of the blade & 60 & $\mathrm{~mm}$ \\
$h$ & Thickness of the blade & 7 & $\mathrm{~mm}$ \\
$k_{n}$ & Normal contact stiffness & $1 \times 10^{5} \sim 1 \times 10^{7}$ & $\mathrm{~N} / \mathrm{m}$ \\
$d$ & Gap between shrouds & $0.02 \sim 0.06$ & $\mathrm{~mm}$ \\
$m_{0}$ & Mass of the shroud & $0.02 \sim 0.1$ & $\mathrm{~kg}$ \\
$n$ & Rotational speed of the blade & $100 \sim 10000$ & $\mathrm{rpm}$ \\
$\mu$ & Rub coefficient & 0.33 & \\
$\gamma$ & Contact angle & $\pi / 3 \sim \pi / 7$ & \\
\hline
\end{tabular}

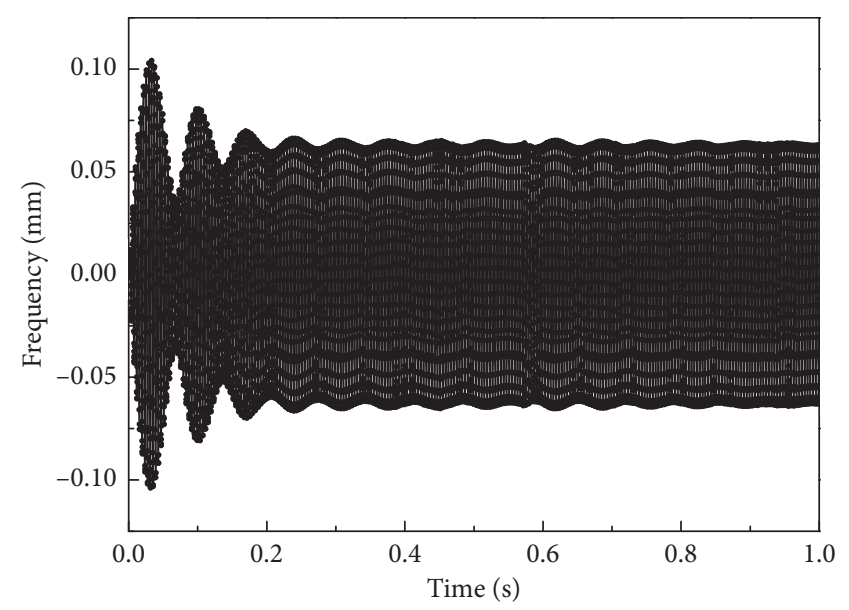

FIgURE 4: Time-domain response of the tip at the rotating speed $5000 \mathrm{rpm}$.

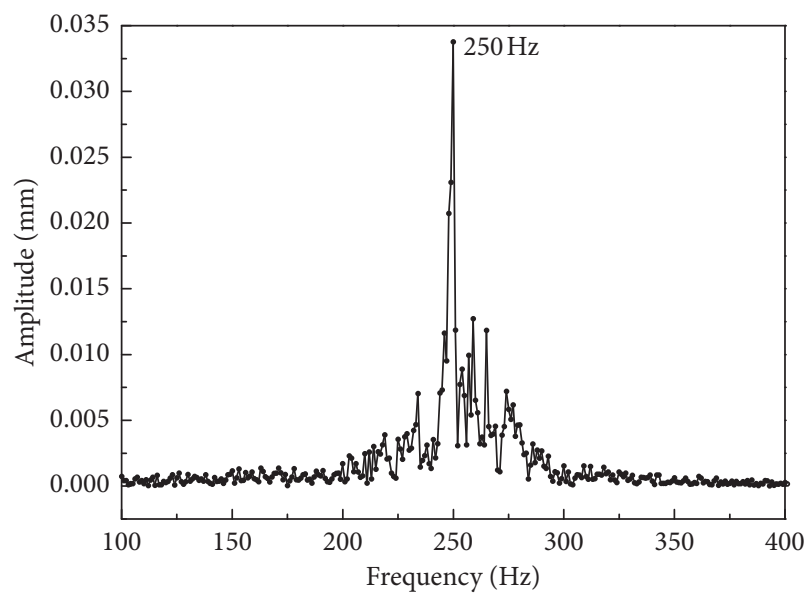

Figure 5: Frequency-domain response of the tip at the rotating speed $5000 \mathrm{rpm}$.

Figure 7 shows the influence of the mass for the shroud on the amplitude of the vibration. The contact angle of the shrouded blade $\gamma$ is equal to $\pi / 3$, the gap of the shroud $d$ is $0.03 \mathrm{~mm}$, and the normal contact stiffness is $1 \times 10^{7} \mathrm{~N} / \mathrm{m}$.

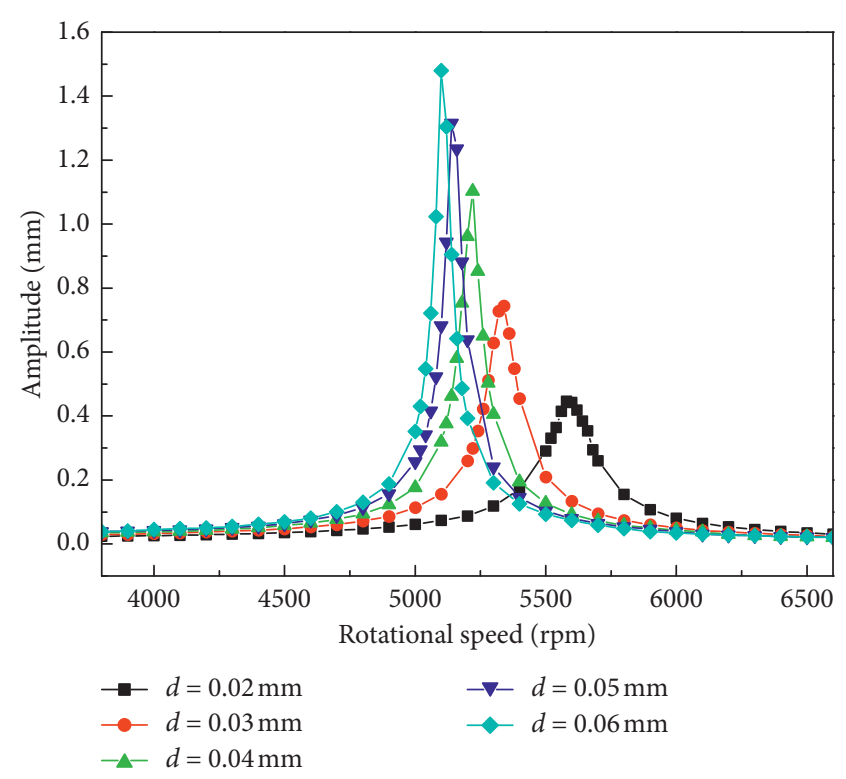

FIgURE 6: The influence of the gap between shrouds on the vibration characteristics.

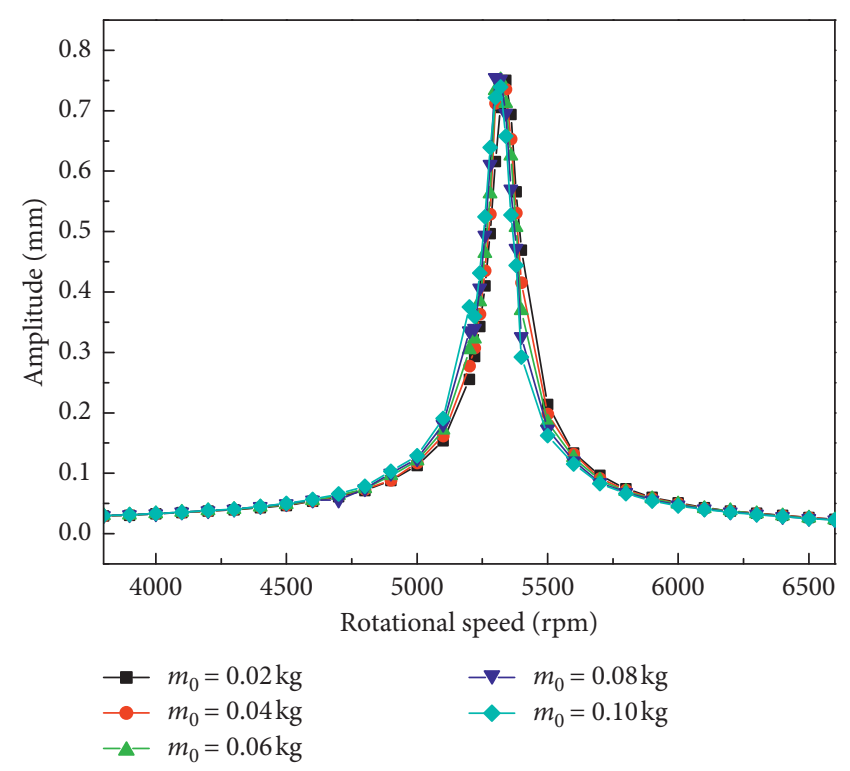

FIgURE 7: The influence of the mass of shrouds on the vibration characteristics.

With the increase of the mass from $0.02 \mathrm{~kg}$ to $0.1 \mathrm{~kg}$, the amplitude of the vibration changes slightly, and the amplitude floats above and below $0.73 \mathrm{~mm}$. It can be seen that the mass of the shroud does not affect the resonant speed. Therefore, in engineering practice, it is useful to design the mass of the shroud to reduce the vibration amplitude without changing the resonant speed.

In order to investigate the influence of the different contact angles for the shroud on the vibration amplitude, the contact angle is given as follows: $\pi / 3, \pi / 4, \pi / 5, \pi / 6$, and $\pi / 7$; the mass of the shroud $m_{0}$ is equal to $0.025 \mathrm{~kg}$; the gap of the shroud $d$ is $0.03 \mathrm{~mm}$; and the normal contact stiffness is $1 \times 10^{7} \mathrm{~N} / \mathrm{m}$. The dynamic response of the system in $Y$ 
direction is calculated. It can be seen from Figure 8 that the vibration amplitude of the blade decreases with the increase of the contact angle. When the contact angle is greater than $\pi / 4$, the change in the vibration amplitude tends to moderate. Equations (5)-(11) show that the contact angle has a great influence on the equivalent stiffness and the damping. Accordingly, increasing the contact angle can effectively reduce the vibration amplitude of the blade. It is also shown from Figure 7 that the mass of the shroud has little effect on the resonant speed.

The normal contact stiffness is related to the material properties of the two contact surfaces, and the range of the normal contact stiffness is from $1 \times 10^{5} \mathrm{~N} / \mathrm{m}$ to $1 \times 10^{7} \mathrm{~N} / \mathrm{m}$ in this study. The mass of the shroud $m_{0}$ is equal to $0.025 \mathrm{~kg}$, the gap of the shroud $d$ is $0.03 \mathrm{~mm}$, and the contact angle of the shrouded blade $\gamma$ is $\pi / 3$. Figure 9 indicates that with the increase of the normal contact stiffness, the amplitude shows decreasing trend. The amplitude of vibration decreases by $62.8 \%$, while the resonance speed increases with increasing of the normal contact stiffness. Hence, one should choose the appropriate material to make the normal contact stiffness as large as possible from the perspective of damping.

\section{Comparison with the Traditional Analytical Method}

The authors studied the effects of the parameters for the shrouded blades on the dynamic characteristics based on the simple Coulomb friction model several years ago. In this paper, based on the new macroslip friction model, the same study has been carried out. The comparison between the traditional method and the new approach will be made. The stiffness has obvious effect on the resonance frequency and the response; with increasing stiffness, the frequency (rotational speed) of the resonance peak increases and the amplitude of the resonance peak decreases. This conclusion based on the new method is the same as that of the traditional method. It validates the correctness of the new approach. The contact angle influences the amplitude obviously and impacts the frequency slightly, especially when the contact angle is greater than $45^{\circ}$. This verifies the new approach from another perspective. The small difference of the other parameters' influence on the dynamics exists in the results. This divergence originates from using of the macroslip friction model, instead of the traditional simple Coulomb friction model. The new approach based on the complex friction model is closer to the reality to get a more accurate calculation. The study is meaningful to the engineering solution and design of the shrouded blades.

\section{Conclusions}

Unlike the traditional analytical method of the shrouded blade based on the simple Coulomb friction model, the macroslip model has been adopted to study the dynamic characteristics of the contact surface for the shrouded blades. Applying the rotational cantilever beam model, the shroud is regarded as a lumped mass and the dynamic equation of the

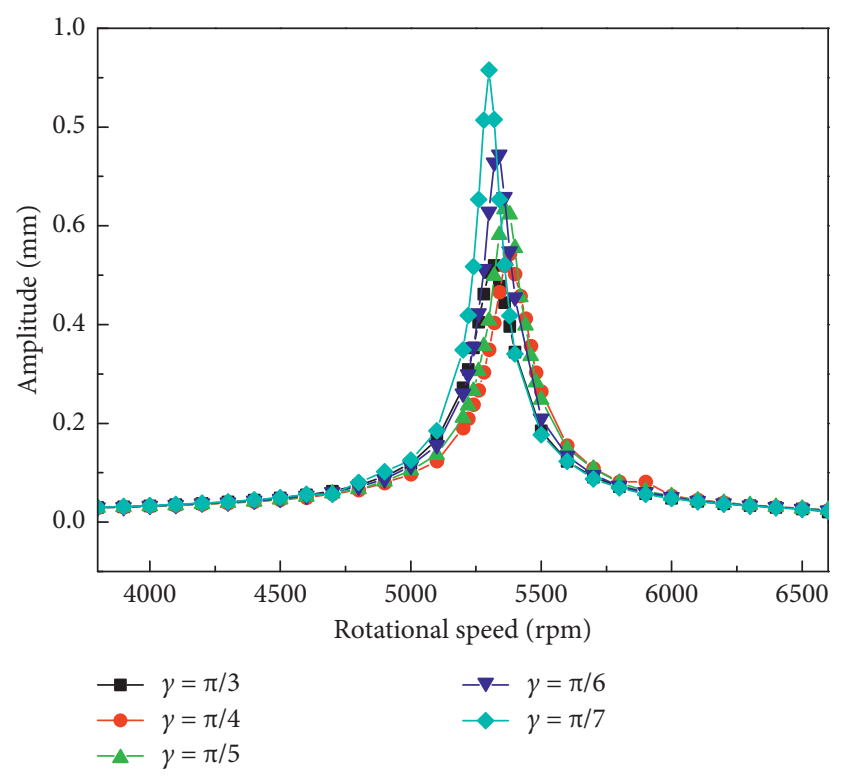

FIGURE 8: The influence of contact angles between shrouds on the vibration characteristics.

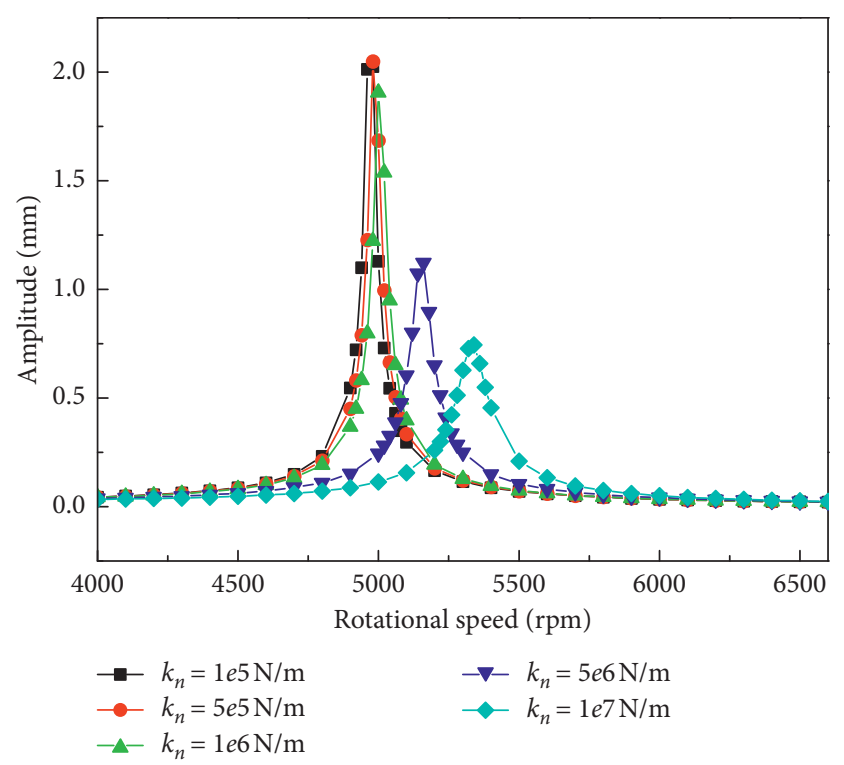

Figure 9: The influence of normal contact stiffness between shrouds on the vibration characteristics.

blade model is obtained to analyze the dynamic characteristics. The effects of parameters such as the gap between the shrouds, the mass of the shroud, the contact angle, and the normal stiffness between the shrouds on the damping characteristics are investigated. Several valuable conclusions are drawn as follows:

(1) The gap has obvious influence on the vibration amplitude of the shrouded blade. The amplitude increases with the increase of the gap (between $0.02 \mathrm{~mm}$ and $0.1 \mathrm{~mm}$ ), while the resonant speed decreases with the increase of the gap. The mass of the shroud is in the range of $0.02 \mathrm{~kg}$ to $0.1 \mathrm{~kg}$ in this 
study. The amplitude of the blade floats above and below $0.73 \mathrm{~mm}$ when the mass is from $0.02 \mathrm{~kg}$ to $0.1 \mathrm{~kg}$, and it does not affect the resonant speed. Therefore, the mass of the shroud has little influence on the vibration frequency.

(2) The contact angle has a great influence on the equivalent stiffness and the damping. Increasing the contact angle can effectively reduce the vibration amplitude. With the increase of the normal stiffness, the vibration amplitude of the blade shows a decreasing trend and the same as the resonant speed. The amplitude of the vibration decreases by $62.8 \%$. The larger normal contact stiffness can effectively reduce the vibration amplitude.

(3) These conclusions are consistent with the trend of the results in published articles [7, 9] and are closer to the conclusion of the paper [9]. This further validates the accuracy of the results based on the new approach. Consequently, the analytical approach based on the macroslip model can effectively solve the contact-impact dynamic responses of the shrouded blades, and the model of the shrouded blades developed in this paper is more accurate than the traditional model based on the traditional simple Coulomb friction. The research work is meaningful to the accurate calculations and design and control of the shrouded blades for rotating machinery.

\section{Nomenclature}

$\begin{array}{ll}X: & \text { The radial direction } \\ Y: & \text { The transverse direction } \\ n: & \text { The rotating speed of the beam } \\ c: & \text { The viscous damping coefficient } \\ x: & \text { The random position along the beam from the } \\ & \text { clamped end } \\ v & \text { The transverse displacement of the beam } \\ (x, t): & \\ v(l): & \text { The transverse displacement at position } v=l \\ \mu: & \text { The friction coefficient between the contact faces } \\ d: & \text { The gap between the shrouds } \\ \gamma: & \text { The contact angle of the shroud } \\ k_{d}: & \text { The shear stiffness of the contact surface } \\ k_{n}: & \text { The normal contact stiffness } \\ m_{0}: & \text { The mass of the shroud } \\ \mathrm{EI}: & \text { The flexural rigidity of the beam } \\ A: & \text { The cross-sectional area } \\ \rho: & \text { The mass density of the blade } \\ l: & \text { The length of the blade } \\ b: & \text { The width of the blade } \\ h: & \text { The thickness of the blade. }\end{array}$

\section{Data Availability}

Some or all data, models, or code generated or used during the study are available from the corresponding author upon request.

\section{Conflicts of Interest}

The authors declare that they have no conflicts of interest.

\section{Acknowledgments}

The authors are grateful to the National Natural Science Foundation of China (Grant no. 51305267).

\section{References}

[1] J. H. Wang and W. K. Chen, "Investigation of the vibration of a blade with friction damper by HBM," Journal of Engineering for Gas Turbines and Power, vol. 115, no. 22, pp. 294-299, 1993.

[2] A. A. Ferri and W. E. Whiteman, "Free response of a system with negative viscous damping and displacement-dependent dry friction damping," Journal of Sound and Vibration, vol. 306, no. 3-5, pp. 400-418, 2007.

[3] R. M. Brach, "Friction, restitution, and energy loss in planar collisions," Journal of Applied Mechanics, vol. 51, no. 1, pp. 164-170, 1984.

[4] J. B. Keller, "Impact with friction," Journal of Applied Mechanics, vol. 3, no. 1, pp. 1-4, 1986.

[5] X.-x. Lu, S.-h. Huang, Z.-q. Liu et al., "Study on contactimpact damping characteristics of shrouded blades based on harmonic balance method," Journal of Power Engineering, vol. 30 , no. 8, pp. 578-583, 2010.

[6] L. Lin, L. Jiu-zhou, and L. Chao, "Review of the dry friction dampers in aero-engine and their design technologies," Journal of Aerospace Power, vol. 31, no. 10, pp. 2305-2317, 2016.

[7] C. H. Menq, J. H. Griffin, and J. Bielak, "The influence of a variable normal load on the forced vibration of a frictionally damped structure," Journal of Engineering for Gas Turbines and Power, vol. 108, no. 2, pp. 300-305, 1986.

[8] R. D. Mindlin, W. P. Mason, T. F. Osmer et al., "Effect of an oscillating tangential force on the contact surfaces of elastic spheres," in Proceedings of the 1st US National Congress of Applied Mechanics, Chicago, IL, USA, June 1951.

[9] A. F. Metherell and R. S. V. Dille, "Instantaneous energy dissipation rate in a lap joint-uniform clamping pressure," Journal of Applied Mechanics, vol. 35, no. 1, pp. 123-128, 1968.

[10] G. F. Nan, X. M. Ren, S. W. He et al., "Damped vibration characteristics of blades with tips of an aero-engine," Journal of Vibration and Shock, vol. 28, no. 7, pp. 135-138, 2009.

[11] G. Nan and Y. Zhang, "Dynamic mechanism and parametric analysis of shrouded blades in aircraft engines," Journal of Vibration Engineering, vol. 17, no. 5, pp. 2132-2142, 1997.

[12] D. Cao, X. Gong, D. Wei, S. Chu, and L. Wang, "Nonlinear vibration characteristics of a flexible blade with friction damping due to tip-rub," Shock and Vibration, vol. 18, no. 1-2, pp. 105-114, 2011.

[13] X.-x. Lu, S.-h. Huang, Z.-q. Liu et al., "The present and progress in the research on impact damping of integrally shrouded blades," Journal of Vibration and Shock, vol. 29, no. 2, pp. 217-218, 2010.

[14] Y. H. Xie and D. Zhang, "Numerical model for vibration characteristics of steam turbine blade with damped shroud," Proceedings of the CSEE, vol. 25, no. 18, pp. 86-90, 2005.

[15] J. Swedowicz, R. Visser, W. Sextro, and P. A. Masserey, "On nonlinear forced vibration of shrouded Turbine Blades," Journal of Turbomachinery, vol. 130, no. 1, pp. 125-128, 2008. 
[16] A. F. Mohammad, A. R. Lopez, and K. Leif, “An analytical calculation of the Jacobian matrix for 3D friction contact model applied to turbine blade shroud contact," Computers \& Structures, vol. 177, no. 12, pp. 204-217, 2016.

[17] H. Ma, F. Xie, H. Nai, and B. Wen, "Vibration characteristics analysis of rotating shrouded blades with impacts," Journal of Sound and Vibration, vol. 378, pp. 92-108, 2016.

[18] F. Xie, H. Ma, C. Cui, and B. Wen, "Vibration response comparison of twisted shrouded blades using different impact models," Journal of Sound and Vibration, vol. 397, pp. 171191, 2017.

[19] B. Wen, H. Ma, X. Yu, J. Zeng, X. Guo, and B. Wen, "Nonlinear vibration and dynamic stability analysis of rotorblade system with nonlinear supports," Archive of Applied Mechanics, vol. 89, no. 7, pp. 1375-1402, 2019.

[20] L. Hou, H. Chen, Y. Chen, K. Lu, and Z. Liu, "Bifurcation and stability analysis of a nonlinear rotor system subjected to constant excitation and rub-impact," Mechanical Systems and Signal Processing, vol. 125, pp. 65-78, 2019.

[21] J. J. Chen and C. H. Menq, "Periodic response of blades having three-dimensional nonlinear shroud constraints," Journal of Engineering for Gas Turbines and Power, vol. 123, no. 4, pp. 901-909, 2001.

[22] A. S. Yigit, A. G. Ulsoy, and R. A. Scott, "Spring-dashpot models for the dynamics of a radially rotating beam with impact," Journal of Sound and Vibration, vol. 142, no. 3, pp. 515-525, 1990. 


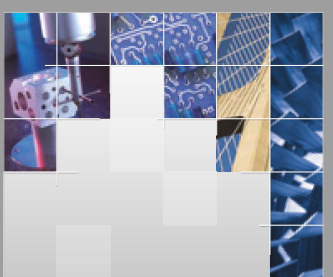

\section{Enfincering}
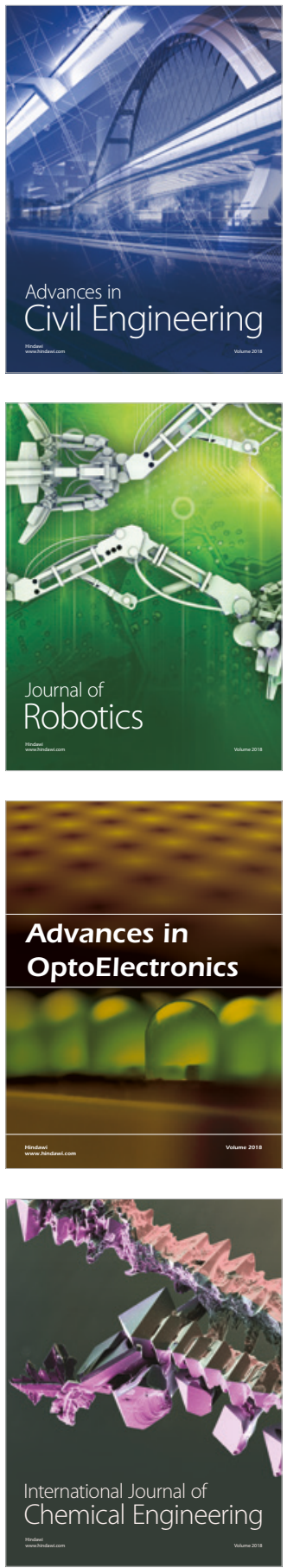

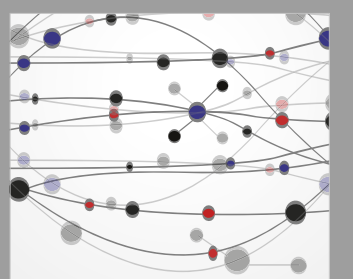

\section{Rotating \\ Machinery}

The Scientific World Journal

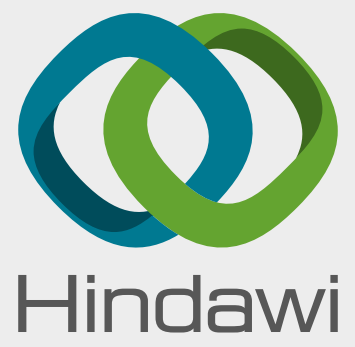

Submit your manuscripts at

www.hindawi.com
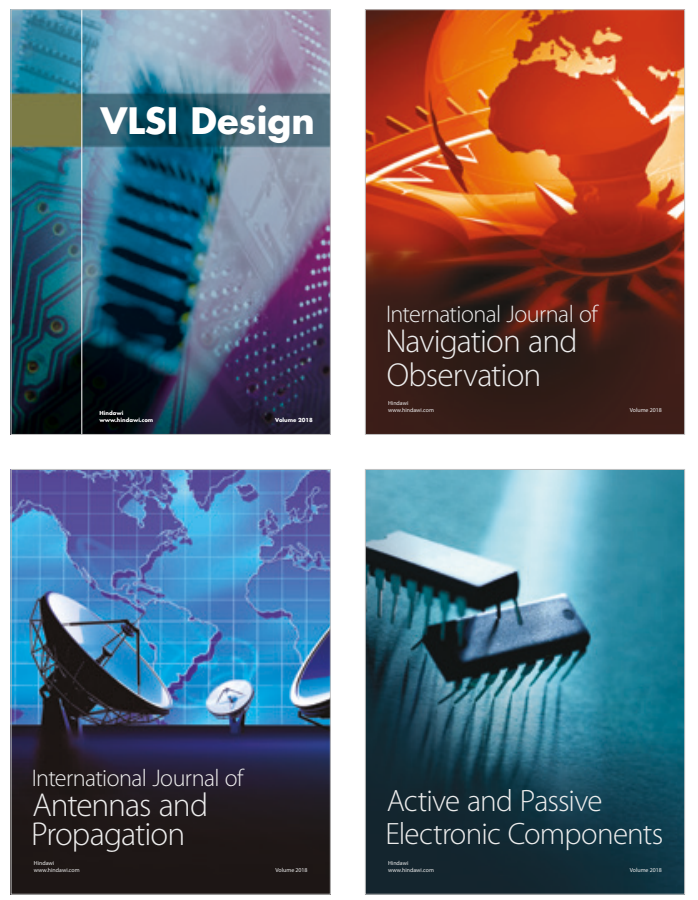
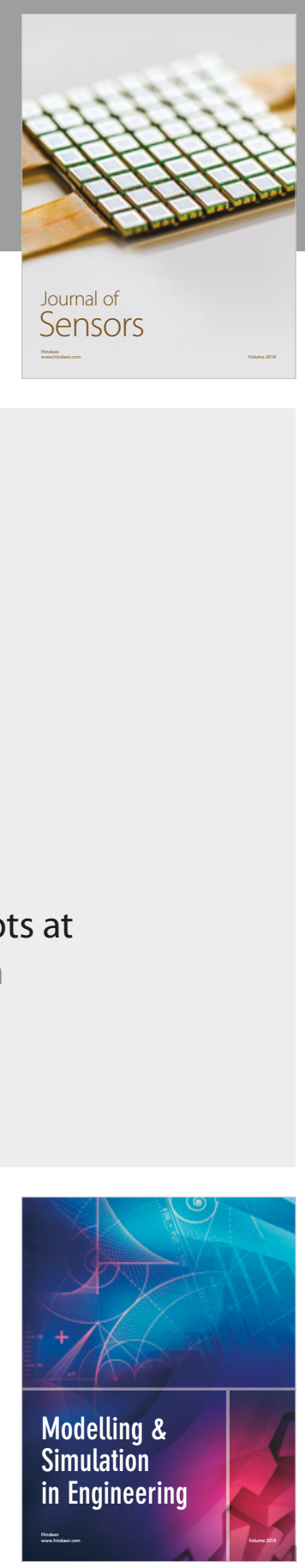

\section{Advances \\ Multimedia}
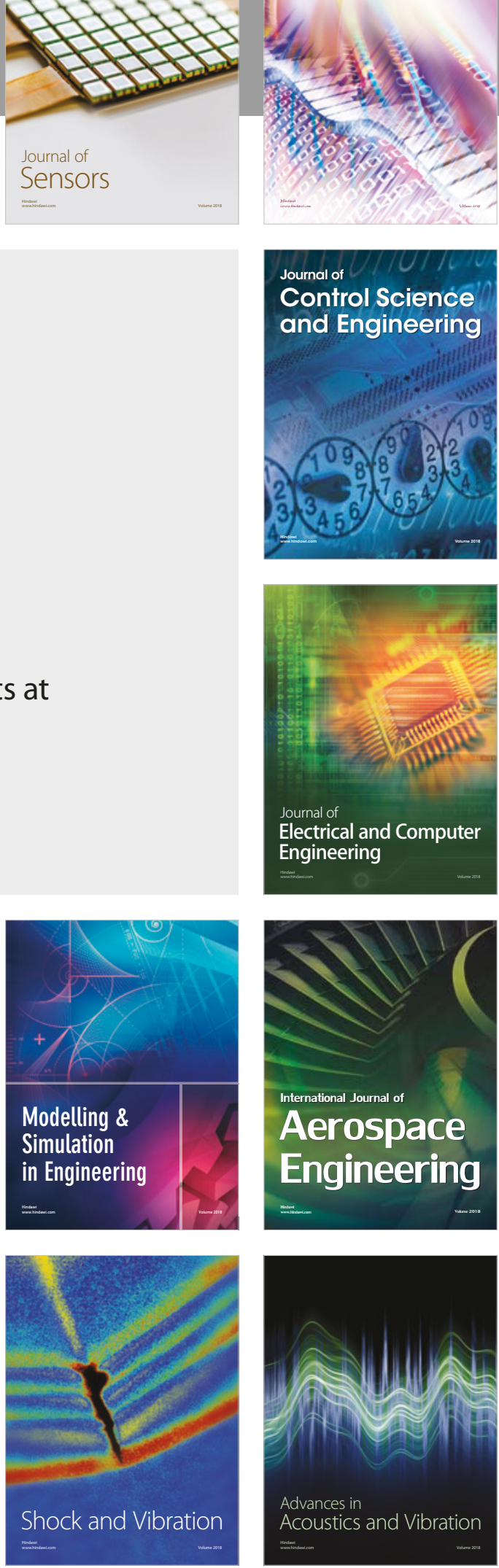